ECONOmía TeORÍA y PrÁctica • Nueva Época, número 47, julio-diciembre 2017, pp. 31-64, http://dx.doi.org/10.24275/ETYPUAM/NE/472017/Verre

\title{
Estrategias de apropiación en contextos de colaboración público-privada en la biotecnología argentina*
}

\section{Appropriation strategies in the context of public-private collaboration in argentine biotechnology}

\author{
Vladimiro Verre, ${ }^{* *}$ Darío Milesi*** \\ y Natalia Petelski****
}

\begin{abstract}
RESUMEN
La actividad innovadora es crítica para una empresa, así como la posibilidad de beneficiarse de sus resultados. Para alcanzar dicho objetivo las firmas elaboran una estrategia de apropiación, basada en el uso de varios mecanismos de apropiación existentes. Este trabajo estudia nueve firmas del sector biofarmacéutico argentino, que se caracteriza por una intensa actividad innovadora que es intrínsecamente cooperativa, ya que las firmas recurren sistemáticamente a fuentes externas y públicas de conocimiento. La colaboración público-privada genera necesariamente efectos sobre las estrategias de apropiación de las empresas, que deben considerar riesgos y ventajas implícitos en un proceso de innovación de tales características. Los rasgos que asume la colaboración entre las partes son críticos para identificar las especificidades de la relación entre colaboración y apropiación, en un sector intensivo en ciencia y tecnología que es estratégico para Argentina por las capacidades acumuladas a nivel científico y empresarial.
\end{abstract}

Palabras clave: Apropiación, innovación, biofarmacéutico, colaboración en I+D, conocimiento Clasificación JEL: O30; O32; O34

\begin{abstract}
Innovative activity is critical to a business, and offers the opportunity to benefit from its results. To achieve this objective, firms develop a strategy of appropriation, based on the use of various existing mechanisms of appropriation. This paper studies nine Argentine firms in the biopharmaceutical sector, which is characterized by intensive innovation, which is inherently cooperative, as firms systematically use external and public sources of knowledge. Public-private partnership necessarily impacts firms' appropriation strategies, which need to consider the risks and benefits inherent to an innovation process of such characteristics. The traits collaboration between the parties assumes are critical to identify the specificities of the relationship between collaboration and appropriation, in a science and technology intensive sector which is strategic for Argentina due to its accumulated scientific and productive capabilities.
\end{abstract}

Keywords: Appropriation, Innovation, Biopharmaceutical, R\&D Cooperation, Knowledge. JEL Classification: O30, O32, O34

* Fecha de recepción: 28/01/2015. Fecha de aprobación: 01/03/2017

** Universidad Nacional de General Sarmiento, Argentina. Correo: vverre@ungs.edu.ar. ORCID 0000-0002-7596-5750

*** Universidad Nacional de General Sarmiento, Argentina. Correo: dmilesi@ungs.edu.ar. ORCID 0000-0002-7596-5750

**** Universidad Nacional de General Sarmiento, Argentina. Correo: npetelsk@ungs.edu.ar. ORCID 0000-0002-2740-4596 


\section{INTRODUCCIÓN}

La competitividad de las firmas y su posicionamiento en mercados locales e internacionales residen cada vez más en su capacidad de innovar. Asimismo, para que la empresa pueda beneficiarse de las innovaciones efectuadas debe estar en condiciones de protegerlas, evitando ser copiada o desplazada del mercado por sus imitadores (Schumpeter, 1942; Arrow, 1962; Teece, 1986). Con el objetivo de proteger su innovación, la firma elabora una estrategia de apropiación, que suele consistir en la articulación de varios mecanismos o acciones. Sin embargo, hay un consenso creciente en la literatura en caracterizar a la innovación como un proceso interactivo y sistémico y, en este marco, una de las situaciones más observadas, particularmente en los sectores "intensivos en ciencia", es la innovación conjunta, entre empresas productivas y fuentes externas de conocimiento, como por ejemplo organismos públicos de investigación y desarrollo (I+D). En este trabajo se plantea que cuando las firmas cooperan para innovar, y particularmente cuando lo hacen con organismos públicos como universidades o centros de $\mathrm{I}+\mathrm{D}$, parte del conocimiento generado queda en poder de actores que, por su propia función, podrían tener como objetivo la difusión del mismo, lo cual implica un riesgo latente para la firma y, por lo tanto, puede afectar su estrategia de apropiación.

En el marco planteado, entonces, el objetivo de este trabajo es analizar los efectos que tiene la colaboración ${ }^{1}$ público-privada (СРP) en I+D sobre la estrategia que la firma desarrolla para apropiarse de los beneficios derivados de las innovaciones generadas en forma conjunta e indagar en qué medida, a diferentes formas de colaboración, corresponden diferentes efectos para la apropiación. Para ello, se analizan nueve firmas argentinas pertenecientes al sector biofarmacéutico, donde una parte importante de la generación del conocimiento se apoya en la CPP.

En la siguiente sección se presenta el marco conceptual que aborda, por una parte, algunos aspectos relevantes de la colaboración en I+D entre actores privados productivos y actores públicos y, por la otra, los mecanismos de apropiación que pueden ser usados por las firmas para diseñar su estrategia de apropiación. A partir de este marco, en la sección 2 se exponen los aspectos metodológicos que caracterizan la investigación realizada y se hace una breve reseña de los casos analizados. Luego, se procede a presentar los resultados obtenidos que abarcan, en la sección 3 , la identificación de diferentes modalidades de colaboración y, en la sección 4, los efectos que generan dichas modalidades de colaboración sobre el uso de los mecanismos de apropiación por parte de las firmas. Finalmente, sobre la base de esa evidencia, en la última sección se plantean las principales conclusiones del trabajo.

'En este artículo se usan los términos “colaboración” y “cooperación” como sinónimos. 


\section{Marco Conceptual}

\section{I.1. La colaboración público-privada en el proceso de innovación}

La literatura que aborda la CPP desde la perspectiva de las firmas se ha concentrado principalmente en el estudio de sus determinantes y de sus efectos sobre el desempeño de las mismas (Tether, 2002; Miotti y Sachwald, 2003; Lööf y Broström, 2008; Belderbos et al., 2004; Eom y Lee, 2009; Ramos-Vielba y Fernández-Esquinas, 2012). Asimismo, otros autores han considerado más detalladamente la multiplicidad de canales existentes a través de los cuales se lleva a cabo la CPP (Bonaccorsi y Piccaluga, 1994; Meyer-Krahmer y Schmoch, 1998; Lee, 1996; Cohen et al., 2000; Schartinger et al., 2002; D’Este y Patel, 2007). Sin embargo, como observan Perkmann y Walsh (2007), estos canales son muy heterogéneos y abarcan desde medios para transmitir la información (publicaciones, patentes) hasta configuraciones y procesos (investigación conjunta, redes). Para comprender la "lógica" de la CPP es necesario entonces analizar también las características que asume la dinámica relacional entre partners y las interacciones que ocurren adentro de la colaboración. Existen algunos estudios orientados a identificar tipologías de colaboración que, sin conformar un marco de análisis común, van en esa dirección y pueden considerarse como un antecedente en este sentido.

Un estudio detallado de la relación universidad-empresa se encuentra en Bonaccorsi y Piccaluga (1994), quienes identifican cuatro dimensiones principales: las motivaciones de la firma para cooperar (acceder al conocimiento científico, reducir los costos de desarrollo o compartir los riesgos, suplir la falta de recursos, etc.), las características del proceso de transferencia de conocimiento (la duración de los proyectos, su proximidad al mercado, la apropiabilidad del conocimiento y las características del conocimiento -cuán tácito es, si es genérico o específico en su aplicabilidad-), la estructura organizativa de la relación (arreglos institucionales), y los procedimientos de coordinación adoptados. Joly y Mangematin (1996), por su parte, se basan en variables como la confianza, el tipo de conocimiento involucrado, la frecuencia de los contratos, el nivel de participación de la firma y el número de partners para identificar tres diferentes lógicas relacionales. Más recientemente, autores como Schartinger et al., (2002) además de considerar el grado de formalización, los recursos asignados por ambos actores, la duración y secuencia de la interacción, han caracterizado los canales de interacción de acuerdo con variables consideradas críticas, tales como la dirección del flujo de conocimiento, la posibilidad de transferir conocimiento tácito y la intensidad de los contactos personales directos. Por su parte, Carayol (2003) se basa en variables como el nivel de riesgo, nivel de novedad, número 
de partners, duración de la colaboración y naturaleza de la investigación (básica/ aplicada) para identificar cinco tipos distintos de colaboración. Bercovitz y Feldman (2007) se basan en el nivel de participación de la firma y las características de los proyectos para distinguir entre dos formas de interacción público-privada: transaccional (proyectos simples y limitados, resultados entregados "llave en mano" a la firma) y relacional (múltiples proyectos, fuertes vínculos entre las partes). Perkmann y Walsh (2007) definen una tipología triádica de vinculación de acuerdo al grado de "involucramiento relacional" entre las partes. Dentro de la categoría de alto involucramiento relacional, los autores distinguen entre "servicios de investigación" (consultorías, contratos de investigación) y "partnership de investigación" (acuerdos formales de investigación conjunta, creación de centros de investigación firma-universidad), según el grado de "finalización" de los proyectos (básico/ aplicado). D'Este y Patel (2007) señalan que diferentes tipos de procesos de transferencia de conocimiento requieren diferentes arreglos interorganizacionales en términos de intercambio de información entre los agentes, recursos involucrados y establecimiento de reglas de apropiación del conocimiento. Levy, Roux y Wolff (2009) cruzan variables como el número de partners, la frecuencia de las interacciones y la variedad de las mismas (publicaciones y patentes conjuntas, contratos espontáneos y subsidiados) e identifican cuatro tipos de colaboración. Arza (2010) identifica una tipología de canales de interacción basada en las motivaciones de los agentes para vincularse y los beneficios alcanzados. En cuanto a las motivaciones de la firma, se distingue entre comportamiento proactivo (interés en acceder al conocimiento y contribuir a generarlo) y pasivo (reducir costos de desarrollo), y en el caso de la universidades entre motivaciones económicas e intelectuales. Los cuatro tipos de colaboración identificados presentan diferencias en relación con variables como la dirección del flujo de conocimiento y el grado de interacción personal entre los actores.

De este modo, la revisión bibliográfica muestra que son diversos los aspectos considerados para caracterizar la CPP, poniendo en evidencia la necesidad de profundizar el estudio hacia adentro de la colaboración para comprender sus características. El siguiente cuadro resume las principales variables mencionadas en los diferentes estudios empíricos y las tipologías de CPP identificadas.

\section{I.2. Colaboración público-privada y apropiabilidad}

Una parte importante de la literatura ha puesto énfasis en las ventajas que representa para las firmas innovar cooperando con instituciones públicas de I+D. Específicamente algunos autores (Cassiman y Veugelers, 2002; Belderbos et al., 2004; 


\section{Cuadro 1. Variables consideradas y tipologías identificadas por la literatura en CPP}

\begin{tabular}{|c|c|c|}
\hline ESTUDIO & VARIABLES CONSIDERADAS & TIPOLOGÍAS \\
\hline $\begin{array}{l}\text { Bonaccorsi y } \\
\text { Piccaluga (1994) }\end{array}$ & $\begin{array}{l}\text { - motivaciones } \\
\text { - duración del proyecto } \\
\text { - } \text { apropiabilidad del conocimiento de conocimiento } \\
\text { - recursos organizacionales aportados } \\
\text { - duración de la interacción } \\
\text { - grado de formalización } \\
\text { - procedimientos de coordinación }\end{array}$ & $\begin{array}{l}6 \text { tipos: relaciones personales informales, re- } \\
\text { laciones personales formales, terceras par- } \\
\text { tes, acuerdos formales focalizados, acuerdos } \\
\text { formales no focalizados, creación de estruc- } \\
\text { turas focalizadas. }\end{array}$ \\
\hline $\begin{array}{l}\text { Joly y Mangematin } \\
\text { (1996) }\end{array}$ & $\begin{array}{l}\text { confianza } \\
\text { - tipo de conocimiento } \\
\text { frecuencia de los contratos } \\
\text { número de partners } \\
\text { participación de la firma }\end{array}$ & $\begin{array}{l}3 \text { tipos: lógica relacional de proximi- } \\
\text { dad, de club, de mercado. }\end{array}$ \\
\hline $\begin{array}{l}\text { Schartinger, et al. } \\
(2002)\end{array}$ & $\begin{array}{l}\text { grado de formalización } \\
\text { posibilidad de transferir conocimiento tácito } \\
\text { - intensidad de contactos personales } \\
\text { dirección del flujo de conocimiento } \\
\text { duración y secuencia de la interacción } \\
\text { motivaciones de la firma } \\
\text { recursos asignados }\end{array}$ & $\begin{array}{l}4 \text { tipos: contratos de investigación, } \\
\text { investigación conjunta, movilidad, ca- } \\
\text { pacitación. }\end{array}$ \\
\hline Carayol (2003) & $\begin{array}{l}\text { nivel de riesgo } \\
\text { - } \text { nivel de novedad } \\
\text { número de partners } \\
\text {. } \text { estructura organizativa } \\
\text {. } \text { duración del proyecto } \\
\text { naturaleza de la investigación }\end{array}$ & $\begin{array}{l}5 \text { tipos (basados en análisis de corres- } \\
\text { pondencia múltiple). }\end{array}$ \\
\hline $\begin{array}{l}\text { Bercovitz y Feldman } \\
\text { (2007) }\end{array}$ & $\begin{array}{l}\text { - nivel de participación de la firma } \\
\text { - duración de los proyectos } \\
\text { - frecuencia de las transacciones } \\
\text {. características del conocimiento }\end{array}$ & 2 tipos: transaccional, relacional. \\
\hline $\begin{array}{l}\text { D'Este y Patel } \\
\text { (2007) }\end{array}$ & $\begin{array}{l}\text { recursos aportados } \\
\text { duración de los acuerdos } \\
\text { grado de formalización }\end{array}$ & $\begin{array}{l}5 \text { tipos: consultorías y contratos de } \\
\text { investigación, investigación conjunta, } \\
\text { capacitación, conferencias, spin-off. }\end{array}$ \\
\hline $\begin{array}{l}\text { Perkmann y Walsh } \\
\text { (2007) }\end{array}$ & $\begin{array}{l}\text { posibilidad de transferir conocimiento tácito } \\
\text {. contactos personales cara a cara } \\
\text {. finalización" (naturaleza de la investigación) }\end{array}$ & $\begin{array}{l}3 \text { tipos: relación, movilidad, transfe- } \\
\text { rencia. }\end{array}$ \\
\hline $\begin{array}{l}\text { Levy, Roux y Wolff } \\
\text { (2009) }\end{array}$ & $\begin{array}{l}\text { número de partners } \\
\text { - frecuencia de la interacción } \\
\text { variedad de las interacciones }\end{array}$ & $\begin{array}{l}4 \text { tipos: relaciones puntuales diádicas; } \\
\text { relaciones puntuales multi-partner; } \\
\text { relaciones regulares multi-partner y } \\
\text { multi-forma; partnerships regulares y } \\
\text { diádicas. }\end{array}$ \\
\hline Arza (2010) & - motivaciones & $\begin{array}{l}4 \text { tipos: servicio, tradicional, comer- } \\
\text { cial, bidireccional. }\end{array}$ \\
\hline
\end{tabular}

Fuente: Elaboración propia con base en la literatura citada. 
López, 2008) señalan que los beneficios para las mismas derivan de la mayor probabilidad de flujos de conocimiento externo captados por la firma (incoming spillovers) en relación con los conocimientos que pueden eventualmente fluir afuera de la firma (outgoing spillovers), en razón de que la contraparte no es un actor de mercado. Bercovitz y Feldman (2007) llegan a conclusiones parecidas, aunque especificando que esta situación es más frecuente en aquellos proyectos que son exploratorios y de largo plazo. Asimismo, la literatura también ha subrayado la existencia de riesgos para la firma a la hora de recurrir a una fuente externa y pública de conocimiento para innovar. Bonaccorsi y Piccaluga (1994), por ejemplo, señalan la existencia de dos riesgos principales; el primero reside en la posibilidad de que los investigadores universitarios se apropien de los resultados de la colaboración y emprendan su propio negocio empresarial; el segundo, que transmitan información útil a los competidores. Los autores resaltan que las patentes pueden ser relativamente inefectivas para enfrentar ambos tipos de riesgos, mientras que otros mecanismos como los activos complementarios o mover primero pueden inhibir el primero de los riesgos, pero no evitan la transferencia (aun involuntaria) de información. Además, la literatura sobre vinculación público-privada coincide en señalar la existencia de diferentes objetivos, normas culturales y prácticas que rigen a la ciencia pública y la investigación privada, entre ellas, difusión y no apropiabilidad versus secreto y apropiabilidad (Cassier, 1997). En la misma línea, Laursen y Salter (2006) señalan la existencia de una paradoja: la generación de la innovación necesita difusión, pero la comercialización de la misma requiere apropiabilidad.

Sin embargo, no es amplia la literatura que analiza la relación entre colaboración público-privada (CPP) y apropiabilidad, y la escasa evidencia empírica al respecto se limita a analizar los efectos del régimen de apropiabilidad sobre la CPP mostrando incluso resultados contradictorios. Mientras algunos autores destacan cómo la gestión de los Derechos de Propiedad Intelectual (DPI) puede ser problemática e inhibir la CPP (Hall et al., 2001; Caloghirou et al., 2001; Lhuillery y Pfister, 2009), otros autores consideran que el régimen de apropiabilidad no es importante para explicar la CPP, dada la naturaleza más genérica del conocimiento involucrado (Cassiman y Veugelers, 2002). Por el contrario, hay autores que subrayan el efecto positivo que los DPI pueden tener para la CPP, por ejemplo, las patentes pueden contribuir a fijar las reglas del juego entre las partes (Brouwer y Kleinknecht, 1999; Arundel, 2001), son fundamentales para evitar problemas de apropiabilidad entre las partes (Schartinger et al., 2002) y facilitan el flujo de conocimiento entre los partners de innovación (Eun et al., 2006).

La revisión bibliográfica pone en evidencia que los estudios que conjugan CPP y apropiabilidad, en general, presentan una de las dos siguientes limitaciones (o las dos a la vez). En primer lugar, considerar a la cPP como una categoría monolítica, es 
decir, no diferenciar entre modalidades de colaboración que pueden variar mucho entre sí, en cuanto a su lógica y sus consecuencias. En segundo lugar, reducir la apropiabilidad a los DPI, sin considerar el más amplio concepto de "estrategia de apropiación". Esta segunda limitación se debe, por un lado, a lo indicado por Perkmann y Walsh (2009) y Perkmann et al. (2013), es decir, una atención predominante de la literatura hacia aquellas formas de colaboración orientadas a la transferencia y comercialización de conocimientos del sector público hacia la firma, donde las patentes y las licencias cumplen un rol central. Por el otro, a una visión según la cual la posibilidad de apropiarse de los resultados de la innovación (y los DPI son considerados un medio determinante y efectivo para tal fin) es la condición que determina ex ante las decisiones de la firma respecto a la innovación (y por ende, a la CPP). En este artículo se parte de una visión diferente, por la cual la estrategia de apropiación, lejos de ser un determinante de la actividad innovadora, es un emergente del proceso de innovación mismo, que se desarrolla generalmente en condiciones de incertidumbre tales que hacen casi imposible prever exante la apropiación de sus resultados (Dosi et al., 2006; Milesi et al., 2013). Los DPI entonces integran, pero no agotan, las distintas acciones o arreglos institucionales que las firmas incorporan al momento de definir su estrategia de apropiación en el contexto de la CPP.

\section{I.3. La estrategia de apropiación de los resultados de la innovación}

Una línea creciente de literatura, iniciada por Levin et al. (1987), se ha centrado en el análisis empírico y conceptual de los mecanismos a través de los cuales las firmas protegen y apropian los beneficios de la innovación. La articulación y combinación de los mecanismos de apropiación disponibles por parte de las firmas es llamada "estrategia de apropiación" (Cohen et al., 2000). De la evidencia recogida en varios trabajos en esta misma línea (Harabi, 1995; Cohen et al., 2000; Arundel, 2001; Galende del Canto, 2006; González y Nieto, 2007, entre otros), se deriva que los principales mecanismos de apropiación pueden clasificarse en dos grandes categorías: i) legales (DPI) y ii) estratégicos.

Los mecanismos de apropiación legales se caracterizan por ser instrumentos formales que implican un registro legal de la innovación. Dentro de este grupo se destaca la patente, que garantiza la apropiación privada a través de los derechos exclusivos que otorga al inventor y permite a su vez cierta difusión del conocimiento al exigir hacer pública la descripción de la innovación (Griliches, 1990). Varios estudios muestran que las patentes son más utilizadas para proteger las 
innovaciones de producto que las de proceso y que su uso y efectividad varían según el sector industrial (Mansfield, 1986; Levin et al., 1987). Entre las limitaciones se destacan la dificultad para demostrar la novedad de la innovación, la revelación de información (disclosure) y los altos costos de aplicación y defensa (Levin et al., 1987; Cohen et al., 2000). Otros mecanismos legales son la licencia (con la cual el innovador cede a otro agente el derecho a la explotación comercial de un DPI, a cambio de regalías), los modelos de utilidad (protegen las mejoras en las prestaciones o funcionalidades de los productos y las innovaciones incrementales), y los modelos/diseños industriales (protegen las características ornamentales, tridimensionales o bidimensionales, de los productos), sobre los cuales no se profundiza al existir escasa evidencia sobre su uso.

Entre los mecanismos de apropiación estratégicos se destacan mover primero, los activos complementarios y el secreto industrial. Mover primero hace referencia al temprano posicionamiento en el mercado de la firma innovadora, el cual le confiere ventajas productivas y comerciales difíciles de igualar por los potenciales competidores. Cuando este comportamiento se repite de forma reiterada, puede considerarse que la firma se apropia de los resultados de su innovación a través de una conducta de "innovación continua", que permite generar periodos sucesivos de exclusividad o liderazgo en el mercado (Fernández Sánchez, 2004; Galende del Canto, 2006). Esta ventaja temporal genera condiciones favorables para la utilización de otros mecanismos tales como la patente o el desarrollo de activos complementarios (Fernández Sánchez, 2004; Milesi et al., 2013). Estos últimos, hacen referencia a un conjunto de capacidades, ventajas o características, que son necesarias para que una innovación sea exitosa en el mercado y el innovador no sea desplazado por sus imitadores (Teece, 1986). Los activos complementarios identificados por la literatura pueden ser agrupados en dos categorías: i) productivos: manufactura competitiva en escala y/o calidad, las tecnologías complementarias, el tipo de equipamiento y el acceso exclusivo a insumo); $i$ ) comerciales: redes de distribución, marketing y servicios postventa ${ }^{2}$ (Milesi et al., 2011). Finalmente, el secreto industrial implica mantener el conocimiento asociado a la innovación dentro de los límites de la empresa. ${ }^{3}$ Es un mecanismo de amplia difusión en la

${ }^{2}$ La posesión de patentes puede funcionar, para la firma, como un activo complementario de índole comercial, según lo indicado por Bureth et al. (2005) y Cimoli y Primi (2008).

${ }^{3}$ La literatura sobre apropiación (Levin et al., 1987; Cohen et al., 2000) considera de manera generalizada a las patentes y al secreto industrial como mecanismos de apropiación alternativos. Otros autores indican que esto no es necesariamente así (Hussinger, 2006; Arundel, 2001; Brouwer y Kleinknecht, 1999), en particular, cuando la innovación recurre a fuentes externas de conocimiento el secreto sigue teniendo una elevada relevancia porque, aun cuando se patente, el proceso de colaboración va generando conocimientos compartidos que pueden ser difundidos y perjudicar a la firma. 
mayoría de los sectores y es más utilizado y más funcional a las innovaciones de proceso (Arundel, 2001; Fernández Sánchez, 2004; González y Nieto, 2007; Galende del Canto, 2006; Harabi, 1995; Milesi et al., 2013). Para mantener la información básica asociada a una innovación en secreto, una empresa puede adoptar medidas respecto a los diferentes actores, por ejemplo, los trabajadores (cláusulas de confidencialidad y políticas de recursos humanos para desincentivar su salida), los proveedores (la integración vertical para evitar difundir especificaciones técnicas clave), los partners de alianzas y acuerdos de colaboración (cláusulas de confidencialidad, establecer condiciones contractuales equitativas que fomenten la confianza y consoliden la interdependencia a largo plazo) (Fernández Sánchez et al., 1998; Hurmelinna-Laukkanen y Puumalainen, 2007).

En relación con la efectividad de los mecanismos, mientras la patente y el secreto industrial están orientados fundamentalmente a evitar la copia de la innovación, los activos complementarios y mover primero apuntan a sostener el posicionamiento en el mercado de la firma aun en el caso de que la innovación sea imitada (Milesi et al., 2013). Dadas las diferentes características y espacios de eficacia de los distintos mecanismos, las firmas suelen utilizarlos simultáneamente y en forma complementaria (Laursen y Salter, 2006). La estrategia de apropiación es, en tal sentido, la articulación que la firma hace de los mecanismos que están a su disposición o que puede generar para proteger su actividad innovadora, cuya configuración depende fuertemente de los rasgos del proceso innovador. En este trabajo se plantea que cuando la innovación se basa en la CPP, la modalidad que asume esta última genera efectos sobre la estrategia de apropiación de la firma: en general, como rasgo estructural del proceso innovador y, en particular, como fuente de riesgo adicional en términos de filtración de conocimiento.

\section{Metodología}

\section{II.1. Selección de los casos}

Por sus características el sector biofarmacéutico argentino constituye un caso relevante en relación con los objetivos de este trabajo. En primer lugar, porque la actividad innovadora es intensiva en colaboración en $\mathrm{I}+\mathrm{D}$ y dicha colaboración es fundamentalmente público-privada (siendo la colaboración firma-firma mucho más débil que en los países desarrollados). En segundo lugar, en un sector relativamente joven y dinámico la heterogeneidad empresarial (incumbents, nuevas empresas, diversas líneas de producto) conlleva heterogeneidad en las modalidades de CPP. En tercer lugar, la importancia sectorial de los DPI (enorme en los países desarrollados) en el contexto local es matizada por estar las empresas 
locales orientadas al mercado de biosimilares, ${ }^{4}$ lo cual permite tomar en cuenta otros aspectos de la estrategia de apropiación a menudo opacados por la relevancia de los DPI a nivel internacional. Adicionalmente, se trata en sí mismo de un caso interesante, ya que no son abundantes los ejemplos de sectores dinámicos e intensivos en $\mathrm{I}+\mathrm{D}$ en países en desarrollo.

Las firmas que utilizan técnicas biotecnológicas en Argentina pertenecientes al sector de salud humana son 26 de acuerdo con datos de 2010 (Gutman y Lavarello, 2010). El trabajo se basa en el estudio de nueve firmas, de capital nacional, cuyas innovaciones biotecnológicas se llevan a cabo en el marco de la CPP. La selección de los casos se realizó con base en información obtenida a través de tres fuentes: estudios sectoriales que analizan la situación de la biotecnología argentina y el comportamiento de sus firmas e instituciones (Bisang et al., 2006; Anlló et al., 2010, entre otros); informantes clave expertos en el sector y conocedores de la relevancia de la actividad innovadora de las firmas; e información institucional de las mismas empresas. Esta selección permitió organizar una base de 13 firmas a contactar, todas ellas localizadas en el área metropolitana de Buenos Aires. De ese grupo, nueve tuvieron disposición para ser entrevistadas y formar parte del presente estudio. Cabe aclarar, sin embargo, que los casos estudiados incluyen dos grupos económicos compuestos por tres y cinco firmas respectivamente y una empresa que controla la propiedad de un start up. Por lo tanto el número de firmas efectivamente abarcado a través de los nueve estudios realizados es de 16, lo cual representa el 60 por ciento del total nacional.

En este marco, se llevó a cabo una investigación exploratoria y descriptiva de naturaleza cualitativa mediante la realización de un estudio de casos múltiples (Eisenhardt 1989; Yin, 1981a, b; Yin, 2003). Los casos seleccionados permiten replicar o extender la teoría emergente (generalización analítica), aunque, como ocurre habitualmente con esta metodología, no se pretende alcanzar generalizaciones estadísticas (Eisenhardt, 1989; Yin, 2003). Por ello, en la selección de los casos se trató de incluir empresas heterogéneas en cuanto a tamaño, antigüedad y líneas de producto (proteínas recombinantes, anticuerpos monoclonales, reactivos de diagnóstico, tejidos, vacunas e insumos) para poder individualizar proyectos de colaboración biotecnológicos que respondieran a diferentes situaciones empresariales, de forma tal

${ }^{4}$ Los biosimilares son segundas versiones de medicamentos o principios activos biotecnológicos que son producidos (con algún grado de innovación en los procesos productivos) y comercializados luego de la expiración de la patente que protege la innovación original (o antes de la misma, en países con sistemas de DPI flexibles). Para que un medicamento sea considerado biosimilar se debe demostrar su similitud con el producto de referencia a través de estudios comparativos de calidad, clínicos y no clínicos. 
que fuera posible identificar diferentes patrones de colaboración y de apropiación, hasta alcanzar la "saturación" de la teoría (Eisenhardt, 1989).

Se partió del marco teórico que orienta la investigación, el cual permitió una preidentificación de los aspectos fundamentales a indagar. El estudio se realizó a través de entrevistas presenciales en profundidad siguiendo una guía de pautas y preguntas abiertas con el presidente de la firma o, en su defecto, con el director de I+D. Las entrevistas se centraron en los aspectos relativos a: la colaboración con instituciones públicas para innovar; la utilización de mecanismos de apropiación, y el impacto de la generación público-privada de conocimiento sobre la estrategia de apropiación. Dada la heterogeneidad de situaciones encontradas, se ha optado por tomar el proyecto biotecnológico, y no la firma, como unidad de análisis, ya que una misma empresa puede llevar adelante varios proyectos que siguen lógicas de colaboración diferentes entre sí. El trabajo aborda el objeto de estudio desde el punto de vista del sector productivo y se centra en las estrategias de apropiación desde la perspectiva de las firmas, por lo que la visión de las instituciones públicas no forma parte del análisis. Además, se han incluido proyectos que están aún en una fase precompetitiva, ya que el foco del trabajo está puesto en la estrategia de apropiación (y los efectos que la colaboración ejerce sobre ella) cuyo carácter intencional y potencial es observable ya en esa fase.

\section{II.2. Los casos estudiados}

Los nueve casos estudiados incluyen a Laboratorio Elea S.A.C.I.F. y A. (Elea), Laboratorios Beta S.A. (Beta), Laboratorio Pablo Cassará S.R.L. (Cassará), Laboratorio Craveri S.A.I.C. (Craveri), Biosidus S.A. (Biosidus), Biocientífica S.A. (Biocientífica), Grupo Amega Biotech (Amega), Delta Biotech S.A. (Delta), y Laboratorio Dosa S.A. (Dosa).

Se trata de una muestra conformada por firmas de distintas edades y tamaños. Cuatro firmas (Elea, Beta, Cassará, Craveri) presentan una trayectoria de más de 60 años en el negocio de los medicamentos. Biosidus y Biocientífica nacieron a inicios de los años 80, mientras que las tres firmas restantes se crearon hacia inicios del 2000. Cabe aclarar que si bien Amega se conformó como Grupo en 2005, las empresas que lo forman tienen una trayectoria previa, ya que datan de los años 90. Los tamaños, tanto en términos de cantidad de empleados como de facturación, se distribuyen de manera similar a la antigüedad, aunque Biocientífica, que no es tan joven, es relativamente pequeña y Amega, que agrupa otras firmas, tiene más empleados que firmas más antiguas (ver cuadro 2). Finalmente, aquellas firmas para las que se cuenta con información muestran una importante orientación hacia el mercado externo. 
Cuadro 2. Información sobre las firmas analizadas

\begin{tabular}{|l|c|c|c|c|}
\hline \multicolumn{1}{|c|}{ FIRMA } & ANTIGÜEDAD & EMPLEADOS & FACTURACIÓN & EXPORTACIONES \\
\hline Laboratorio Elea & 1939 & 800 & 185 Mill. USD & ND \\
\hline Laboratorios Beta & 1940 & 700 & 74 Mill. USD & $0 \%$ (BIO) \\
\hline Laboratorio Craveri & 1942 & 300 & 30 Mill. USD & ND \\
\hline Laboratorio Pablo Cassará & 1948 & 500 & $25-30$ Mill. USD & $20 \%(10 \%$ BIO) \\
\hline Biosidus & 1983 & 370 & 40 Mill. USD & $80 \%$ \\
\hline Biocientífica & 1983 & 50 & ND & $20 \%$ \\
\hline Laboratorio Dosa & 1999 & 50 & ND & $40 \%(0 \%$ BIO) \\
\hline Delta Biotech & 2003 & 25 & $3-4$ Mill. USD & $50 \%$ \\
\hline Amega Biotech & 2005 & 230 & 10 Mill. USD & $75 \%$ \\
\hline
\end{tabular}

ND: No disponible.

Fuente: Elaboración propia con base en datos de 2012.

Si bien todas las firmas desarrollan proyecto de I+D en el área biofarmacéutica en colaboración con centros públicos, las capacidades propias, las áreas específicas en las que se desempeñan y las modalidades a través de las cuáles cooperan difieren.

Entre los casos estudiados, Elea ${ }^{5}$ es la firma más antigua. Se trata también de una de las empresas farmacéuticas más antiguas del país, con una larga trayectoria en endocrinología, hormonas y piojicidas. La experiencia desarrollada en el manejo de proteínas le permite interesarse tempranamente en la biotecnología y su adquisición por el Grupo Chemo en los años 90 determina la profundización de las actividades biotecnológicas. Entre tales actividades se destacan: el desarrollo de vacunas oncológicas basadas en gangliósidos en colaboración con centros de investigación cubanos y argentinos, varios desarrollos en el ámbito oncológico en colaboración con la Universidad Nacional de Quilmes (UNQ) y el desarrollo de una planta para la producción de anticuerpos monoclonales. Lo que caracteriza a tales proyectos es el co-desarrollo, es decir, son llevados a cabo por una serie de instituciones públicas y privadas (LANAIS-CITO, Instituto Roffo, Instituto Nacional de Tecnología Industrial -INTI-, PharmAdn) que actúan como un consorcio y donde se trabaja en red. Dicha red se basa en una división del trabajo articulada y las instituciones públicas que participan en la misma (tanto centros de I+D como hospitales) realizan tareas que van desde las investigaciones básicas hasta la etapa clínica. Entre ellas, la institución pública que cumple un papel central en la generación del conocimiento es el Instituto de Oncología Molecular de la UNQ.

${ }^{5}$ Para el caso de Elea, perteneciente al Grupo Chemo, ver Gutman y Lavarello (2010). 
Beta tiene casi la misma antigüedad que Elea y cuenta con una larga trayectoria en antiinflamatorios, antirreumáticos y productos hormonales. Su actividad principal es la producción de insulina. Su incursión en la biotecnología parte del intento de desarrollar un proyecto para producir insulina recombinante humana. Inicialmente enfrentó limitaciones en las capacidades internas que la indujeron a buscar el apoyo del Instituto de Biología y Medicina Experimental (IByME), con el que ya existían colaboraciones previas. Con el avance del proyecto, la empresa finalmente decide incorporar en relación de dependencia a un grupo de investigadores biotecnólogos que trabajan en dicha institución. Si bien los trabajadores pasan a ser personal de la firma, continúan trabajando físicamente en el IByмE, lo cual les permite seguir investigando en un ámbito académico y nutrirse del conocimiento que circula en el mismo. A partir de 1996 la firma realiza varias innovaciones de proceso, consolida el proyecto y extiende su campo de acción a otros productos afines, como los análogos de insulina, dando inicio a la producción en 2006.

Craveri, fundada en 1942, se especializa actualmente en tejidos humanos y piel. En 1996 crea la División de Bioingeniería, un emprendimiento biotecnológico orientado a la ingeniería de tejidos (generación de sustitutos biológicos que restauran o mejoran la función de órganos o tejidos dañados). La firma tiene relaciones de colaboración de larga data con el Instituto Leloir en temas de piel y cuando decide entrar en la ingeniería de tejidos, incorpora en relación de dependencia a un grupo de investigadores de aquel Instituto, que con el tiempo logran realizar innovaciones que permiten a la firma ampliar la gama de tejidos producidos. Esto posicionó a Craveri como la única empresa nacional productora de tejidos, donde cuenta con una cartera de alrededor de 10 productos en distintas fases de desarrollo. Más recientemente, la firma emprendió otro proyecto con el Instituto Leloir, una vacuna oncológica basada en la terapia génica. Algunas fases de este proyecto se basan en una división del trabajo, otras son realizadas conjuntamente, en el marco de constantes interacciones y de resultados compartidos.

Cassará también tiene una larga trayectoria en la producción de medicamentos, siendo pionero en aerosoles antiasmáticos. En 1984 su presidente crea la Fundación Cassará, un centro de I+D que hoy tiene importantes vínculos con el sector público (en ella trabajan investigadores del Consejo Nacional de Investigaciones Científicas y Técnicas - Conicet- y funciona el Centro Milstein, creado conjuntamente con el Conicet). Los primeros proyectos biotecnológicos del Laboratorio se llevan a cabo en colaboración con la Fundación, que es la que tiene el know how en esa área. El Laboratorio tiene una alianza estratégica con la Fundación y ha emprendido numerosos proyectos con ella: producción de proteínas recombinantes (fue la segunda empresa después de Biosidus en desarrollarlas), vacunas contra hepatitis, anticuerpos monoclonales, entre otros. La flexibilidad de los límites entre la Fundación y la firma, 
hace que ésta se encuentre en un lugar privilegiado para detectar nuevas ideas, seleccionar proyectos a desarrollar en distintas áreas del conocimiento y fomentar el surgimiento de start ups. Los proyectos siguen la lógica del co-desarrollo y la titularidad de las patentes biotecnológicas generadas no es de la firma sino de los start ups que surgen, aunque la firma suele ser socia mayoritaria de estos últimos.

De fundación más reciente que las firmas descriptas previamente, Biosidus ${ }^{6}$ representa uno de los casos más estudiados del área biotecnológica en Argentina y Sudamérica. Se trata de una empresa que desde sus comienzos se orientó a producir proteínas recombinantes, logrando una cartera de siete proteínas. En tal sentido, constituye un caso único en América Latina que se basa tanto en la creación de capacidades internas de $\mathrm{I}+\mathrm{D}$ como en la colaboración de prestigiosos científicos de instituciones públicas. En la segunda mitad de los 90 Biosidus entra en el campo de la clonación y del desarrollo de animales de granja como biorreactores (utilización de animales para que produzcan en su leche proteínas recombinantes como la insulina o la hormona del crecimiento humano). El proyecto del "tambo farmacéutico" se inicia en colaboración con el Instituto de Ingeniería Genética y Biología Molecular (INGEBI) y el IBYME (fase I), pero posteriormente la firma opta por llevarlo adelante internamente, incorporando a importantes recursos humanos de aquellas instituciones públicas que habían participado de la primera fase del proyecto y apoyándose en los centros públicos de I+D para servicios críticos (fase II). En 1999 Biosidus emprende, junto a la Fundación Favaloro, un proyecto sobre terapia génica, que consiste en la inserción de copias funcionales de genes defectuosos o ausentes en el genoma de un individuo, en este caso para favorecer la revascularización. El proyecto se basa en una división del trabajo horizontal, ya que Biosidus se dedica a la construcción genética y a la presentación de las investigaciones clínicas a las entidades regulatorias, mientras que la Fundación Favaloro se enfoca en los ensayos sobre animales, la investigación clínica y el suministro a pacientes. Los resultados son compartidos y las patentes generadas son de titularidad conjunta.

Biocientífica también fue fundada en los años 80 , pero tiene menor tamaño que Biosidus a la vez que se orienta a un segmento de menor complejidad científico-tecnológica que esta última. En tal sentido, Biocientífica se especializa en reactivos para el diagnóstico de enfermedades humanas en tres áreas: autoinmunidad, enfermedades infecciosas y proteínas plasmáticas de interés clínico. A partir de la segunda mitad de la década del 2000 comienza a usar técnicas de ADN recombinante. El mercado de estos productos es un nicho donde prácticamente no hay competidores y sus clientes son hospitales, centros de investigación y laboratorios

${ }^{6}$ Para el caso de Biosidus, ver Aguiar (2011). 
de análisis clínico. La firma coopera con varios centros públicos de I+D, como el INTI y la Facultad de Farmacia y Bioquímica de la Universidad de Buenos Aires (FFyB-UBA), a los que contrata la realización de servicios relacionados con su actividad productiva tales como el control de calidad sobre insumos químicos usados, la realización de ensayos sobre animales en bioterios ${ }^{7} \mathrm{y}$ el desarrollo de productos puntuales.

Entre las empresas de creación más reciente se destaca Amega, ${ }^{8}$ que en muy pocos años desde su fundación ha logrado alcanzar un tamaño mediano y orientar su negocio preferentemente al mercado externo. Esta empresa tiene el centro de su actividad en la producción de proteínas recombinantes biosimilares y posee importantes capacidades internas de I+D. Pero además cuenta con fuentes externas de conocimiento a las que recurre regularmente, siendo la Universidad Nacional del Litoral (UNL) la más relevante. Una de las firmas del Grupo, Zelltek, fue el primer start up surgido de una universidad argentina (la UNL), actualmente se especializa en la producción de eritropoyetina (EPO) y tiene su equipo de I+D localizado en el mismo espacio físico del Laboratorio de Cultivos Celulares de la Facultad de Bioquímica y Ciencias Biológicas de la UNL (LCC-UNL). Esto genera un ambiente amplio, mixto y prolífico en generación y circulación de conocimientos, donde hay cierta flexibilidad en los límites entre la empresa y la institución. En el marco de dicha alianza estratégica se llevan a cabo numerosos proyectos que incluyen proteínas recombinantes, anticuerpos monoclonales y vacunas. En estos proyectos se sigue un esquema de codesarrollo y se comparten los resultados, lo cual se refleja en la cotitularidad de las patentes generadas.

$\mathrm{Al}$ igual que Amega, Delta ${ }^{9}$ ha logrado un rápido crecimiento aunque se mantiene de todas maneras como una firma pequeña. Produce principios activos de alta potencia (calcitrón y prostaglandinas) para el mercado de medicamentos genéricos, con un alto nivel de valor agregado (algunos ingredientes valen $U$ \$D 70,000 por gramo). La actividad se lleva a cabo en un nicho de mercado con muy pocos competidores a nivel internacional. Con la aplicación de técnicas biotecnológicas, la firma ha realizado importantes innovaciones de proceso que la han vuelto muy competitiva, logrando un rápido crecimiento y la extensión de dichas innovaciones a otros productos afines. Delta realiza el desarrollo del proceso en parte internamente y en parte externamente, apoyándose en los vínculos de su presidente con el ámbito científico. El desarrollo del proceso es tercerizado a partir de su separación en distintos bloques de etapas de síntesis química, para lo cual la firma acude a

\footnotetext{
${ }^{7}$ Lugar en el que se crían, mantienen y utilizan animales de laboratorio.

${ }^{8}$ Para el caso de Amega Biotech, ver Gutman y Lavarello (2010).

${ }^{9}$ Para el caso de Delta, ver Verre et al. (2014).
} 
diversos actores como la UBA, el Centro de Excelencia en Productos y Procesos de la Provincia de Córdoba (Ceprocor), la Universidad de Amsterdam, entre otros.

Finalmente, Dosa, fundada en 1999, se dedica a fabricar medicamentos genéricos tradicionales y desde 2004 incursiona en la biotecnología, a través de un contrato de colaboración con la Universidad Nacional de Quilmes (UNQ), para identificar un proceso productivo innovador para la producción del filgrastim (molécula recombinante de uso oncológico). Una vez finalizado el proyecto, se solicita una patente a nombre de la firma y los conocimientos son transferidos de la UNQ a la firma, que posteriormente emprende la fase de escalado industrial en colaboración con el INTI.

El cuadro 3 sintetiza las principales características de los casos estudiados en lo relativo a los proyectos biotecnológicos que han sido considerados en este estudio, al partner público involucrado en cada caso y a las formas de colaboración utilizadas.

Cuadro 3. Información sintética sobre los proyectos analizados

\begin{tabular}{|c|c|c|c|}
\hline FIRMA & $\begin{array}{c}\text { PROYECTOS } \\
\text { BIOTECNOLÓGICOS }\end{array}$ & PARTNER PÚBLICO & FORMA DE COOPERACIÓN \\
\hline Laboratorio Elea & $\begin{array}{l}\text { Vacunas oncológicas; anti- } \\
\text { cuerpos monoclonales }\end{array}$ & $\begin{array}{l}\text { UNQ, LANAIS-CITO, Insti- } \\
\text { tuto Roffo, INTI, otros }\end{array}$ & Codesarrollo \\
\hline Laboratorios Beta & $\begin{array}{l}\text { Insulina recombinante hu- } \\
\text { mana; análogos de insulina }\end{array}$ & IByME & $\begin{array}{l}\text { Incorporación de personal } \\
\text { del centro público que sigue } \\
\text { trabajando físicamente en el } \\
\text { centro }\end{array}$ \\
\hline Laboratorio Craveri & $\begin{array}{l}\text { Ingeniería de tejidos; vacuna } \\
\text { oncológica }\end{array}$ & Instituto Leloir & $\begin{array}{l}\text { Incorporación de personal } \\
\text { del centro público. } \\
\text { Codesarrollo }\end{array}$ \\
\hline $\begin{array}{l}\text { Laboratorio Pablo } \\
\text { Cassará }\end{array}$ & $\begin{array}{l}\text { Proteínas recombinantes; va- } \\
\text { cunas, anticuerpos monoclo- } \\
\text { nales }\end{array}$ & $\begin{array}{l}\text { Fundación Cassará, Coni- } \\
\text { cet }\end{array}$ & Codesarrollo \\
\hline Biosidus & $\begin{array}{l}\text { Proteínas recombinantes; } \\
\text { tambo farmacéutico; } \\
\text { terapia génica }\end{array}$ & $\begin{array}{l}\text { Consultores científicos; } \\
\text { IByME, INGEBI; } \\
\text { Fundación Favaloro }\end{array}$ & $\begin{array}{l}\text { Incorporación de personal } \\
\text { del centro público. Contra- } \\
\text { tación de servicios críticos. } \\
\text { Codesarrollo }\end{array}$ \\
\hline Biocientífica & Reactivos de diagnóstico & INTI, FFyB-UBA & Contratación de servicios \\
\hline Laboratorio Dosa & Filgrastim & UNQ & Contratación de desarrollos \\
\hline Delta Biotech & $\begin{array}{l}\text { Principios activos de alta po- } \\
\text { tencia }\end{array}$ & Ceprocor, FFyB-UBA. & Contratación de desarrollos \\
\hline Amega Biotech & $\begin{array}{l}\text { Proteínas recombinantes, an- } \\
\text { ticuerpos monoclonales; va- } \\
\text { cunas. }\end{array}$ & LCC-UNL & $\begin{array}{l}\text { Codesarrollo. Equipo de I+D } \\
\text { de la empresa trabajando fí- } \\
\text { sicamente en centro público } \\
\text { de I+D }\end{array}$ \\
\hline
\end{tabular}

Fuente: Elaboración propia. 


\section{LA IDENTIFICACIÓN DE DIFERENTES ESQUEMAS DE COLABORACIÓN}

El análisis de los casos pone de manifiesto que la colaboración de las firmas con las instituciones públicas se desarrolla de formas variadas. En el extremo, se podría afirmar que cada caso presenta particularidades. Sin embargo, también se observan algunas recurrencias o patrones de interacción que permiten la identificación de una tipología de esquemas de colaboración. La literatura revisada en la primera sección indica la existencia de un conjunto de variables que son cruciales para caracterizar a la CPP e identificar tipologías. En este estudio, se ha optado por estilizar la CPP desde la perspectiva de las firmas y a partir de cuatro parámetros que, como muestra el cuadro 1 presentado previamente, se encuentran entre los más recurrentemente utilizados en estudios similares. Para cada parámetro es posible especificar el rango de variación observado en los casos estudiados, de la siguiente forma: $i$ ) el objetivo de la colaboración ${ }^{10}$ (complementación de capacidades existentes, internalización de capacidades públicas y generación conjunta de nuevas capacidades); ii) el grado de participación directa de la firma en las actividades de I+D ${ }^{11}$ (de bajo -servicio recibido "llave en mano"- a alto -codesarrollo-); iii) la duración del vínculo ${ }^{12}$ (de corto plazo a largo plazo); iv) la frecuencia de las interacciones ${ }^{13}$ (de baja a alta). Este enfoque se sintetiza en la figura 1.

Los casos estudiados presentan diversidad de situaciones en los cuatro parámetros considerados. En lo relativo al objetivo se observa que las empresas más pequeñas (Dosa, Biocientífica y Delta) acuden a los organismos públicos para complementar las capacidades existentes en la firma. En estos casos, los organismos con los que se vinculan aportan servicios puntuales en actividades o fases en las que las firmas no tienen capacidad interna de I+D. En el otro extremo, los casos de Elea, Amega, Cassará, Biosidus para el tambo farmacéutico fase I y Craveri para la vacuna oncológica, se vinculan con los institutos públicos de $\mathrm{I}+\mathrm{D}$ para emprender proyectos conjuntos con un mayor grado de incertidumbre en los que tanto la firma como el

\footnotetext{
${ }^{10}$ El parámetro es central en el análisis de Bonaccorsi y Piccaluga (1994) y Arza (2010); también es considerado por Schartinger et al. (2002).

${ }^{11}$ Este parámetro es clave en el análisis de Bercovitz y Feldman (2007) y está presente en el de Joly y Mangematin (1996); Schartinger et al. (2002) y Arza (2010) en términos de flujos de conocimiento.

${ }^{12}$ El parámetro ocupa un lugar importante en Bonaccorsi y Piccaluga (1994); es considerado también por D'Este y Patel (2007); Carayol (2003); Bercovitz y Feldman (2007); Schartinger et al. (2002).

${ }^{13}$ Este parámetro es crítico en Bonaccorsi y Piccaluga (1994), Schartinger et al. (2002) y Perkmann y Walsh (2007) en relación con las interacciones "cara a cara"; también es considerado en Levy, Roux y Wolff (2009) y Bercovitz y Feldman (2007).
} 
Figura 1. Parámetros utilizados para caracterizar la colaboración desde la perspectiva de la firma

\begin{tabular}{|lcc|}
\hline & $\mathrm{DE}$ & $\mathrm{A}$ \\
Objetivo & Complementación & Generación conjunta \\
Participación & Bajo & Elevado \\
Duración & Corto plazo & Largo plazo \\
Frecuencia & Baja & Alta \\
\hline
\end{tabular}

Fuente: Elaboración propia.

partner público aportan a la generación conjunta de conocimientos y capacidades. En una situación intermedia, los casos Beta, Biosidus para la producción de proteínas recombinantes y el tambo farmacéutico fase II y Craveri para ingeniería de tejidos, muestran el objetivo de incrementar las capacidades de la firma a partir de la internalización de las capacidades públicas, objetivo que materializan a través de la incorporación a la firma de los investigadores del sector público que participan en los proyectos de I+D emprendidos en forma conjunta. La frecuencia de la interacción es creciente a medida que se pasa de vínculos centrados en un servicio específico a los otros que involucran objetivos más complejos. Lo mismo ocurre con la duración del vínculo.

Con respecto a la participación de la firma, los casos de Dosa, Delta y Biocientífica muestran un involucramiento reducido de las mismas en las actividades de I+D objeto del vínculo con los institutos públicos. Las firmas solicitan servicios o desarrollos que los institutos realizan y entregan prácticamente "llave en mano" sin una intervención importante de las mismas. En el mejor de los casos, las firmas complementan internamente estas actividades, pero casi sin interacción con los institutos públicos. Un grado mayor de participación se observa en los casos en los que las firmas terminan incorporando los recursos humanos públicos a su staff como se observa en Beta, Biosidus para las proteínas recombinantes y el tambo farmacéutico fase II y Craveri para ingeniería de tejidos. Aquí se da una evolución de la participación de la firma en un doble sentido. Por un lado, la firma se involucra progresivamente en las actividades de $\mathrm{I}+\mathrm{D}$ en la medida en que, al incorporar los recursos humanos públicos, se hace cargo crecientemente de las 
mismas. Por el otro, la incorporación a la firma de los recursos humanos del instituto público de $\mathrm{I}+\mathrm{D}$ puede implicar una merma de la frecuencia de la interacción con el mismo porque las capacidades que antes eran provistas desde el instituto están ahora integradas a la firma. Sin embargo, en los casos estudiados, tanto por el hecho de no modificar la localización física de estos recursos (caso Beta) como por las vinculaciones personales que los mismos mantienen con colegas que permanecen en sus instituciones de origen, el vínculo de la firma con los institutos públicos se ha mantenido o incluso fortalecido. Finalmente, en los proyectos más complejos en los que el conocimiento y las capacidades son generadas conjuntamente como se observa en el caso de Elea, Amega, Cassará, Biosidus para tambo farmacéutico fase I y terapia génica, y el caso de Craveri para la vacuna oncológica, el involucramiento y la participación de la firma en las actividades de $\mathrm{I}+\mathrm{D}$ se mantiene elevada y con un rol de la firma en la generación del nuevo conocimiento que es equivalente y, en algunos casos, superior al del organismo público con el que coopera.

En este marco, desde la perspectiva de las firmas, los casos analizados permiten identificar tres esquemas de colaboración: contratación, internalización y coordinación. Tales esquemas en parte se encuentran en línea con la dicotomía que emerge de la literatura revisada, entre transacción y relación (Bercovitz y Feldman, 2007), flujos bidireccionales y monodireccionales de conocimiento (Meyer-Krahmer y Schmoch, 1998; Arza, 2010), partnership y servicios de investigación (Perkmann y Walsh, 2007), academic engagement y comercialización (Perkmann et al., 2013). Asimismo, también dan cuenta de una modalidad de colaboración, la internalización, que presenta características propias e intermedias, en línea con lo identificado por Perkmann y Walsh (2007). ${ }^{14}$ El cuadro 4 sintetiza los esquemas identificados y su caracterización en términos de los parámetros utilizados.

Habiendo identificado y caracterizado estilizadamente los esquemas a través de los cuales las firmas estudiadas cooperan con los centros públicos de I+D, el siguiente paso consiste en analizar el impacto de la colaboración público-privada sobre la estrategia desarrollada por las firmas para apropiar los conocimientos generados.

\footnotetext{
${ }^{14}$ Cabe señalar que en la categoría "movilidad” identificada por Perkmann y Walsh (2007), así como ocurre en la "internalización", los investigadores públicos que se mueven hacia la industria suelen mantener lazos con sus ex colegas académicos. Sin embargo, en la "internalización" se observa que lo que las firmas absorben no son simplemente investigadores públicos individuales, sino grupos completos de investigación que trabajan en instituciones públicas de I+D.
} 
50 ECONOMÍA TEORÍA Y PRÁCTICA • Nueva Época, número 47, julio-diciembre 2017

Cuadro 4. Esquemas de colaboración en los casos estudiados

\begin{tabular}{|c|c|c|c|}
\hline PARÁMETRO & CONTRATACIÓN & INTERNALIZACIÓN & COORDINACIÓN \\
\hline Objetivo & $\begin{array}{l}\text { Complementar capaci- } \\
\text { dades }\end{array}$ & Generar capacidades internas & Generar capacidades conjuntas \\
\hline $\begin{array}{l}\text { Participación de } \\
\text { la firma }\end{array}$ & $\begin{array}{l}\text { Escasa, servicios " llave } \\
\text { en mano", división del } \\
\text { trabajo rígida }\end{array}$ & $\begin{array}{l}\text { Creciente en la actividad de I+D } \\
\text { (aumenta con la incorporación de } \\
\text { RRHH externos) }\end{array}$ & $\begin{array}{c}\text { Alta, codesarrollo, división del } \\
\text { trabajo basada en la interacción } \\
\text { constante }\end{array}$ \\
\hline $\begin{array}{l}\text { Extensión en el } \\
\text { tiempo }\end{array}$ & $\begin{array}{l}\text { Corto plazo: realización } \\
\text { de fases específicas }\end{array}$ & Proyectos complejos de largo plazo & $\begin{array}{c}\text { Proyectos complejos de largo } \\
\text { plazo }\end{array}$ \\
\hline Frecuencia & Baja-media & $\begin{array}{c}\text { Media (disminuyen las interacciones } \\
\text { con la incorporación de RRHH ex- } \\
\text { ternos) }\end{array}$ & Alta \\
\hline Casos & $\begin{array}{c}\text { Dosa; } \\
\text { Delta; } \\
\text { Biocientífica }\end{array}$ & $\begin{array}{l}\text { Beta; } \\
\text { Biosidus: proteínas recombinantes, } \\
\text { tambo farmacéutico fase II; } \\
\text { Craveri: ingeniería de tejidos }\end{array}$ & $\begin{array}{c}\text { Elea; } \\
\text { Amega; } \\
\text { Cassará; } \\
\text { Biosidus: tambo farmacéutico } \\
\text { fase I, terapia génica; } \\
\text { Craveri: vacuna oncológica }\end{array}$ \\
\hline
\end{tabular}

Fuente: Elaboración propia.

\section{Colaboración Y ESTRATEgIA DE APROPIACIÓN}

\section{Las firmas estudiadas utilizan los distintos mecanismos de apropiación reportados por la literatura de manera complementaria, como se observa en el cuadro 5.}

Cuadro 5. Uso de mecanismos de apropiación en los casos estudiados

\begin{tabular}{|c|c|c|c|c|}
\hline \multirow[b]{2}{*}{ EMPRESA } & \multirow[b]{2}{*}{ MECANISMOS LEGALES } & \multicolumn{3}{|c|}{ MECANISMOS ESTRATÉGICOS } \\
\hline & & SECRETO & $\begin{array}{l}\text { MOVER } \\
\text { PRIMERO }\end{array}$ & $\begin{array}{c}\text { ACTIVOS } \\
\text { COMPLEMENTARIOS }\end{array}$ \\
\hline Amega & $\begin{array}{l}\text { Patentes defensiva } \\
\text { en Interferón y } \\
\text { EPO, ofensivas en } \\
\text { Células Vero }\end{array}$ & $\begin{array}{l}\text { Contratos confi- } \\
\text { dencialidad; } \\
\text { control sobre los } \\
\text { start ups }\end{array}$ & $\begin{array}{l}\text { Ampliación de la gama de } \\
\text { PR; múltiples proyectos } \\
\text { innovadores (vacunas, an- } \\
\text { ticuerpos monoc., etc.) }\end{array}$ & $\begin{array}{l}\text { Grupo MegaPharma; imagen } \\
\text { de la firma }\end{array}$ \\
\hline Beta & $\begin{array}{l}\text { Patentes defensivas } \\
\text { (alguna ofensiva en } \\
\text { análogos de insuli- } \\
\text { na) }\end{array}$ & $\begin{array}{l}\text { Contratos confi- } \\
\text { dencialidad; } \\
\text { incorporación } \\
\text { de RRHH }\end{array}$ & $\begin{array}{l}\text { Primer productor de insu- } \\
\text { lina recombinante en Ar- } \\
\text { gentina, análogos de in- } \\
\text { sulina }\end{array}$ & $\begin{array}{l}\text { Imagen/trayectoria de la fir- } \\
\text { ma; red comercial; servicio al } \\
\text { cliente }\end{array}$ \\
\hline Biocientífica & NO & $\begin{array}{l}\text { Contratos confi- } \\
\text { dencialidad }\end{array}$ & $\begin{array}{l}\text { Ampliación de la gama de } \\
\text { reactivos (nicho) }\end{array}$ & $\begin{array}{l}\text { Imagen de la firma; } \\
\text { certificaciones de calidad UE }\end{array}$ \\
\hline
\end{tabular}




\begin{tabular}{|c|c|c|c|c|}
\hline \multirow[b]{2}{*}{ EMPRESA } & \multirow[b]{2}{*}{ MECANISMOS LEGALES } & \multicolumn{3}{|c|}{ MECANISMOS ESTRATÉGICOS } \\
\hline & & SECRETO & $\begin{array}{l}\text { MOVER } \\
\text { PRIMERO }\end{array}$ & $\begin{array}{c}\text { ACTIVOS } \\
\text { COMPLEMENTARIOS }\end{array}$ \\
\hline Biosidus & $\begin{array}{l}\text { Patentes defensivas } \\
\text { en proteínas, ofen- } \\
\text { sivas en clonación, } \\
\text { terapia génica }\end{array}$ & $\begin{array}{l}\text { Contratos confi- } \\
\text { dencialidad; } \\
\text { incorporación } \\
\text { de RRHH }\end{array}$ & $\begin{array}{l}\text { Primer productor de PR en } \\
\text { ARG y ampliación de la } \\
\text { gama; múltiples proyectos } \\
\text { innovadores (tambo } \\
\text { farm., terapia génica, clo- } \\
\text { nación de caballos, } \\
\text { Agro-Biotechnology, etc.) }\end{array}$ & $\begin{array}{l}\text { Grupo Sidus; } \\
\text { Imagen/trayectoria de la fir- } \\
\text { ma; red de distribución; re- } \\
\text { gulatorios; servicio al cliente; } \\
\text { patentes }\end{array}$ \\
\hline $\begin{array}{l}\text { Laboratorio } \\
\text { Cassará }\end{array}$ & $\begin{array}{l}\text { Patentes defensivas } \\
\text { en proteínas, ofen- } \\
\text { sivas en vacunas }\end{array}$ & $\begin{array}{l}\text { Contratos confi- } \\
\text { dencialidad; } \\
\text { control sobre los } \\
\text { start ups }\end{array}$ & $\begin{array}{l}\text { Ampliación de la gama de } \\
\text { PR; múltiples proyectos } \\
\text { innovadores (vacunas) }\end{array}$ & $\begin{array}{l}\text { Imagen de la firma; } \\
\text { Red de ventas; patentes }\end{array}$ \\
\hline $\begin{array}{l}\text { Laboratorio } \\
\text { Craveri }\end{array}$ & $\begin{array}{l}\text { Patente ofensiva en } \\
\text { vacuna, defensivas } \\
\text { en tejidos }\end{array}$ & $\begin{array}{l}\text { Contratos confi- } \\
\text { dencialidad; } \\
\text { incorporación } \\
\text { de RRHH }\end{array}$ & $\begin{array}{l}\text { Ampliación de la gama } \\
\text { tejidos (nicho); varios pro- } \\
\text { yectos innovadores (tera- } \\
\text { pia génica) }\end{array}$ & $\begin{array}{l}\text { Imagen de la firma; } \\
\text { patente }\end{array}$ \\
\hline Delta Biotech & NO & $\begin{array}{l}\text { Contratos confi- } \\
\text { dencialidad; } \\
\text { fragmenta el co- } \\
\text { nocimiento }\end{array}$ & $\begin{array}{l}\text { Ampliación de la gama de } \\
\text { algunos principios activos } \\
\text { (nicho) }\end{array}$ & $\begin{array}{l}\text { Imagen de la firma; alianza } \\
\text { con firmas UE; tipo de equi- } \\
\text { pamiento; certificación de } \\
\text { calidad }\end{array}$ \\
\hline $\begin{array}{l}\text { Laboratorio } \\
\text { Dosa }\end{array}$ & Patente defensiva & $\begin{array}{l}\text { Contratos confi- } \\
\text { dencialidad }\end{array}$ & NO & $\begin{array}{l}\text { Amplio portafolio de produc- } \\
\text { tos }\end{array}$ \\
\hline $\begin{array}{l}\text { Laboratorio } \\
\text { Elea }\end{array}$ & $\begin{array}{l}\text { Patentes ofensivas } \\
\text { en oncología }\end{array}$ & $\begin{array}{l}\text { Contratos confi- } \\
\text { dencialidad; } \\
\text { confianza en la } \\
\text { red de múltiples } \\
\text { actores }\end{array}$ & $\begin{array}{l}\text { Múltiples proyectos inno- } \\
\text { vadores (oncología, anti- } \\
\text { cuerpos monoc., vacuna } \\
\text { gripe H1N1, etc.) }\end{array}$ & $\begin{array}{l}\text { Grupo Chemo; imagen/ tra- } \\
\text { yectoria de la firma; red de } \\
\text { distribución; } \\
\text { regulatorios }\end{array}$ \\
\hline
\end{tabular}

Fuente: Elaboración propia.

Analizando por cada mecanismo, se puede apreciar que la mayoría de las empresas utiliza mecanismos legales, principalmente la patente, aunque se observan dos situaciones distintas en lo relativo a su carácter: hay patentes "defensivas" y "ofensivas". Las primeras protegen innovaciones puntuales en nichos de conocimiento que han quedado vacantes. Suele ser el caso de patentes sobre innovaciones de proceso, que la firma solicita para proteger los procedimientos con los que trabaja y, de ese modo, evitar que terceras empresas la demanden por copia y detengan su actividad productiva. Tales patentes suelen tener por único titular a la firma y se pueden observar en el caso de las proteínas recombinantes biosimilares (Biosidus, Amega, Beta y Dosa) o en las patentes que Craveri tiene intención de solicitar en el ámbito de los tejidos. El segundo caso, las patentes "ofensivas", se presenta cuando las innovaciones alcanzan mayor envergadura y el patentamiento persigue el objetivo de evitar la copia y proteger una innovación potencialmente rentable en el mercado. Dichas patentes pueden observarse en Biosidus, para el tambo farmacéutico y 
la terapia génica, y en distintos proyectos inherentes a vacunas, como en los casos de Elea, Craveri, Amega y Cassará. En estos casos puede haber cotitularidad público-privada o titularidad exclusivamente pública de la patente, aunque en este último caso la parte pública suele ceder en licencia exclusiva la explotación comercial de la patente a la parte privada, a cambio de regalías sobre ventas futuras.

En lo relativo al secreto, como ya se mencionó, cuando una firma recurre a una fuente externa y pública para generar nuevo conocimiento queda expuesta a riesgos de filtraciones. Por ejemplo, los recursos humanos públicos que la firma no controla y participan del proceso de generación pueden divulgar información clave para la firma, o bien, ingresar a una empresa competidora o dar inicio a un start up, que puede transformarse en un nuevo competidor (Bonaccorsi y Piccaluga, 1994). Este riesgo derivado de la CPP es reconocido por todas las firmas estudiadas. Sin embargo, ante dichos riesgos, las firmas analizadas responden haciendo un uso más complejo del secreto que el que reporta la literatura. En tal sentido, el análisis del uso del mecanismo del secreto en los casos estudiados permite identificar dos dimensiones principales: una pasiva y la otra activa.

La primera se refiere a factores contextuales que desincentivan eventuales comportamientos oportunistas del partner público que pongan en peligro la efectividad del secreto industrial. En primer lugar, la naturaleza de las instituciones con las que se coopera que, al ser públicas y académicas, hace menos probable que las mismas decidan orientarse a una experiencia empresarial y productiva. En segundo lugar, quien decida eventualmente crear una nueva firma competidora debe enfrentar importantes barreras a la entrada, como la necesidad de efectuar costosas inversiones y de contar con activos complementarios críticos, entre ellos el know how empresarial. En tercer lugar, hay en general un importante nivel de confianza en la contraparte que, en algunos casos, responde a relaciones personales construidas en el tiempo, pero en otros, refleja la existencia de relaciones más formales y articuladas.

Los factores contextuales mencionados sirven a su vez de apoyo a la segunda dimensión del uso del secreto, la activa, que consiste en medidas y comportamientos que la empresa decide tomar para controlar información valiosa y evitar que la misma se filtre hacia competidores actuales o potenciales. En primer lugar, se destacan los contratos y las cláusulas de confidencialidad, que son instrumentos muy difundidos en los casos estudiados. Los contratos suelen describir detalladamente de quién es la propiedad del conocimiento previo y del que se genera durante la colaboración. Sin embargo, en general, las empresas usan este instrumento con escasa seguridad acerca de su eficacia real.

Otro modo de fortalecer el secreto consiste en la incorporación de recursos humanos que provienen de las instituciones públicas con las que se coopera. Desde esta óptica puede considerarse la incorporación de investigadores del IByME, 
por Laboratorios Beta en la insulina recombinante y por Biosidus en el tambo farmacéutico, y del Instituto Leloir, por Laboratorio Craveri. La incorporación de esos recursos humanos puede responder a dos objetivos paralelos: por un lado, crear capacidades en la firma internalizando la fuente externa de conocimiento y, por el otro, evitar que el conocimiento generado quede fuera del control la firma. De esta forma, la firma endogeniza el riesgo, trasladando el problema del secreto hacia el ámbito que controla.

Una tercera forma de mantener el secreto frente a las instituciones públicas de I + D consiste en la fragmentación del conocimiento. Es el caso de Delta Biotech, cuyo proceso productivo innovador consiste de numerosas etapas de síntesis quími$\mathrm{ca}$, algunas de las cuales son desarrolladas internamente, mientras que en otras se encarga su desarrollo a varios centros públicos de $\mathrm{I}+\mathrm{D}$, de modo que ninguna institución conozca la secuencia completa del proceso innovador.

Una cuarta forma de fortalecer el uso del mecanismo del secreto es estrechando alianzas estratégicas con el partner público. Cuando la colaboración evoluciona desde meras colaboraciones esporádicas y ad hoc a proyectos complejos de codesarrollo puede entreverse la existencia de una alianza estratégica entre la firma y la institución pública, que puede abarcar un proyecto biotecnológico concreto (Biosidus) o múltiples proyectos (Laboratorio Pablo Cassará, Laboratorio Elea y Amega Biotech). En estos proyectos de largo plazo se suelen generar patentes de titularidad compartida entre las partes, lo cual contribuye a generar un clima de confianza que tiene efectos sobre el conocimiento protegido a través del secreto, ya que en el marco de dicha alianza crecen los incentivos a la confianza mutua y a evitar comportamientos oportunistas, en línea con lo observado por Arundel (2001) y Hussinger (2006). Este aspecto se diferencia de la confianza de la dimensión pasiva, en la que se apoyan todas las firmas estudiadas (y que constituye una condición mínima para la existencia de la colaboración), ya que representa su fortalecimiento formal y temporal.

Finalmente, un modo adicional de fortalecer el mecanismo del secreto consiste en la vigilancia sobre la generación de conocimiento de centros de I+D. En el caso de Amega Biotech y Laboratorio Pablo Cassará hay una importante circulación de recursos humanos entre la empresa y el partner público, se comparte el mismo espacio físico y hay cierta superposición entre los recursos humanos. Esta configuración hace que, cuando se genera nuevo conocimiento que está relacionado o es complementario al que ya posee la firma (o cuando la situación de su propiedad es ambigua), la firma puede, en cierta forma, anticiparse a la salida de ese conocimiento, evitando que fluya hacia un competidor ya existente o hacia un nuevo competidor (como un start up desvinculado de la firma), lo cual pondría en riesgo conocimientos críticos. Las firmas mencionadas logran detectar con rapidez la presencia de vocaciones emprendedoras y proyectos atractivos, apoyándolos y encauzándolos 
por ejemplo mediante la incubación de start ups de los que luego se vuelven socias mayoritarias.

En las firmas estudiadas también se verifica un uso generalizado tanto de la estrategia de mover primero como de algunos activos complementarios $\mathrm{y}$, en muchos casos, es posible detectar cómo la colaboración público-privada representa para la firma un apoyo fundamental en la implementación de dichos mecanismos.

Respecto a mover primero, este mecanismo puede abarcar tanto innovaciones incrementales como radicales y su uso apunta a objetivos diversos y complementarios, como penetrar nichos de mercado vacantes, disputar la cuota de mercado a quien fuera el único productor o ampliar la gama de productos de la empresa. Beta es un laboratorio antiguo con una larga trayectoria innovadora que se caracteriza por el uso de este mecanismo y que, gracias al aporte público del IByME, consolida dicho comportamiento en el área biotecnológica, concentrándose en la insulina recombinante y en nuevos productos ligados a la misma, como los análogos de insulina. Elea y Craveri también tienen una larga trayectoria y su ingreso en la biotecnología está ligado a productos estratégicos y de largo plazo, como la vacuna oncológica con la UNQ y los tejidos con el Instituto Leloir, respectivamente. Ambas han emprendido proyectos posteriores, por ejemplo, Elea en anticuerpos monoclonales, y Craveri en terapia génica y nanotecnología, con notable participación del sector público. No obstante ser una empresa relativamente nueva y pequeña, Delta basa su estrategia en la ampliación de la gama de los principios activos que produce y puede sustentarla gracias a la red de proveedores públicos que ha logrado conformar. Biosidus, Amega y Cassará han perseguido el mismo objetivo que Delta, ampliar la gama, pero en productos más complejos como las proteínas recombinantes, apoyándose respectivamente en consultores científicos pertenecientes a instituciones públicas, en la UNL y en la Fundación Cassará. Estas tres empresas, además de ampliar la gama de proteínas biosimilares, han llevado adelante una multiplicidad de proyectos biotecnológicos, algunos de importante nivel de novedad, con distintas instituciones públicas de I+D. Entre dichos proyectos se puede mencionar, por ejemplo, los de las áreas de vacunas, anticuerpos monoclonales y nanotecnología (casos Amega y Cassará), y la vasta serie de proyectos altamente innovadores de Biosidus, que van desde la terapia génica con la Fundación Favaloro, al tambo farmacéutico (que se alimentó de conocimientos provenientes del IByME) y otros numerosos proyectos orientados a aprovechar sus plataformas tecnológicas. Por lo tanto, en todos los casos es posible apreciar cómo la CPP se convierte en parte constitutiva fundamental de la estrategia de mover primero que las firmas pueden desarrollar.

Algo similar se observa en lo referido a los activos complementarios. En este aspecto, la CPP representa para las firmas un factor que contribuye a crear o consolidar activos importantes para ser usados como mecanismo de apropiación. En 
cuanto a los productivos, puede citarse el caso de Delta, donde el tipo de equipamiento es un activo complementario clave, ya que la innovación de proceso se traduce en una planta piloto con características técnicas únicas que representa un factor crítico para que el proceso asuma las cualidades que lo destacan. En la medida en que el desarrollo de la innovación se realiza a través de una red de subcontrataciones parciales entre centros públicos de I+D, la colaboración desempeña un rol clave en la creación de este activo. Asimismo, las fuentes externas y públicas de conocimiento pueden representar un apoyo importante para algunos activos complementarios comerciales. En primer lugar, uno de los activos complementarios comerciales más relevantes suele ser la imagen de la firma y, en los casos de las empresas de mayor dimensión como Biosidus, Elea y Amega, resulta claro que la colaboración contribuye al fortalecimiento de ese activo. Un caso emblemático es Biosidus, cuyo renombre internacional también se debe a la participación de prestigiosas instituciones públicas en varios de sus proyectos, lo cual se refleja en la existencia de patentes conjuntas y en el apoyo dado por investigadores públicos durante el lanzamiento en el mercado de algunos productos de la firma. En segundo lugar, la patente puede funcionar como un activo complementario comercial y favorecer tanto la imagen de la firma como su poder de negociación (Bureth et al., 2005; Cimoli y Primi, 2008), lo cual es observable en casos como Craveri, Elea, Cassará y Biosidus. Finalmente, la CPP permite a las firmas fortalecer activos complementarios de índole regulatoria, ya que la aprobación y el registro de medicamentos son influenciados positivamente por la calidad y el prestigio de las instituciones en las que se llevan a cabo los estudios clínicos. Puede destacarse, por ejemplo, el rol positivo que cumplen para Elea instituciones públicas prestigiosas como el Instituto Roffo y el LANAIS-CITO para los estudios clínicos sobre la vacuna oncológica. En el caso de Biosidus, los estudios clínicos efectuados sobre proteínas recombinantes (por ejemplo, en el caso de la EPO) fueron dirigidos por destacados científicos de la UBA, lo cual no solamente fue importante desde el punto de vista regulatorio, sino que además permitió a Biosidus estrechar lazos con la comunidad médica que resultaron claves para que la firma ganara la confianza de los médicos y tuviera mayor llegada al mercado.

La evidencia analizada permite también notar que, además de los efectos generales de la colaboración sobre la estrategia de apropiación, existen efectos específicos derivados de las particularidades de cada esquema de colaboración. En lo relativo a patentes, el esquema de coordinación suele generar titularidades compartidas, lo cual implica menor valor relativo apropiable para la firma, pero sobre patentes ofensivas, basadas en desarrollos novedosos y con potencialidad para apropiar muy relevantes cuotas de valor, una vez en el mercado. Considerando el esquema de internalización, se observa que suele generar patentes defensivas de las que la 
firma es titular exclusiva y cuya principal contribución es evitar juicios y ahorrar tiempo y recursos (una excepción es constituida por las patentes ofensivas de Biosidus en el tambo farmacéutico). Finalmente, el esquema de contratación de servicios puntuales no parece estar orientado a generar DPI relevantes y cuando hay patentes, como en el caso de Dosa, éstas suelen ser de carácter defensivo, por lo que su contribución a evitar la copia y a la apropiación es limitada.

En el caso del secreto, si bien las firmas analizadas se apoyan tanto en su dimensión pasiva como en los contratos de confidencialidad dentro de los tres esquemas de colaboración identificados, los esquemas difieren en su potencialidad para favorecer la adopción de aquellas medidas complementarias que las firmas pueden emplear para complejizar el uso de este mecanismo. Dicha complejización corresponde, en el esquema de contratación, a la fragmentación del conocimiento (el caso de Delta Biotech) y en el esquema de internalización, a la absorción de recursos humanos públicos (con el doble objetivo de crear capacidades y proteger el conocimiento en ellos incorporado). Estos aspectos inherentes a la dimensión activa del secreto, conllevan algún grado de reducción de los alcances de la CPP, contrariamente al caso del esquema de coordinación, donde la confianza en la contraparte es fortalecida en un marco de alianza estable y con reglas claras, que además de ofrecer un contexto favorable a una colaboración más intensa, permite la adopción de medidas creativas, tales como vigilar y controlar la generación de start ups, aprovechando la emergencia de eventuales nuevos proyectos.

En lo relativo a los restantes mecanismos estratégicos, el esquema de contratación da un apoyo limitado a mover primero, que se vuelve más relevante cuanto mayor es la frecuencia de la colaboración, como en el caso de la ampliación de la gama de productos de Delta Biotech. Los activos complementarios no reciben en este esquema un apoyo decisivo, aunque se puede considerar el conocimiento y el acceso a los partners públicos como parte de los principales activos complementarios de estas firmas. El apoyo que reciben dichos mecanismos parece ser más sustancial en firmas que adoptan esquemas de colaboración más complejos, como la internalización y la coordinación. En el esquema de internalización la colaboración tiene un efecto positivo en la estrategia de mover primero (ampliación de la gama de proteínas y tejidos, en los casos de Biosidus y Craveri respectivamente) y en el fortalecimiento de un activo crítico como la imagen de la firma. La coordinación, por su parte, parece ser el esquema más adecuado para maximizar los efectos positivos de la colaboración sobre estos mecanismos estratégicos generando, por un lado, un importante caudal de proyectos innovadores que enriquecen el mover primero, y por el otro, fortaleciendo varios activos complementarios de envergadura, como por ejemplo la imagen de la firma, los activos regulatorios y el portafolio de patentes, que además de ser un reflejo del mover primero, funcionan 
como activos complementarios. Los diferentes efectos que genera cada esquema de colaboración sobre el uso de los distintos mecanismos de apropiación se sintetizan en el cuadro 6.

Como se mencionó, estos efectos distintivos se dan en el marco de dos fenómenos generales. Por un lado, en todos los esquemas el secreto se ve desafiado, aunque el carácter no competitivo de los partners públicos (la denominada aquí "dimensión pasiva") mitiga en alguna medida el inevitable riesgo de filtraciones implicado en la colaboración en comparación con lo que ocurriría si la colaboración se diera con potenciales competidores en el mercado. Por el otro, en todos los esquemas hay un efecto positivo de la colaboración sobre mover primero, activos complementarios y el uso de mecanismos legales, aunque la dimensión y relevancia de este efecto varía considerablemente de un esquema a otro. Los diferentes esquemas de colaboración se diferencian entonces no sólo en relación con la magnitud de dicho efecto positivo (sobre mover primero, activos complementarios y mecanismos legales), sino también en el hecho de otorgar distintos márgenes de acción a la firma para implementar medidas u acciones para atenuar el efecto negativo de la CPP sobre el secreto.

Cuadro 6. Efectos de los esquemas de colaboración sobre el uso de mecanismos de apropiación

\begin{tabular}{|l|l|l|l|}
\hline \multicolumn{1}{|c|}{ MECANISMO } & \multicolumn{1}{|c|}{ CONTRATACIÓN } & \multicolumn{1}{c|}{ INTERNALIZACIÓN } & \multicolumn{1}{c|}{ COORDINACIÓN } \\
\hline Secreto industrial & $\begin{array}{l}\text { 1. Dimensión pasiva } \\
\text { 2. Contratos de confiden- } \\
\text { cialidad } \\
\text { 3. Fragmentación del cono- } \\
\text { cimiento }\end{array}$ & $\begin{array}{l}\text { 1. Dimensión pasiva } \\
\text { 2. Contratos de confiden- } \\
\text { cialidad } \\
\text { 3. Incorporación de recursos } \\
\text { humanos públicos }\end{array}$ & $\begin{array}{l}\text { 1. Dimensión pasiva } \\
\text { 2. Contratos de confidencialidad } \\
\text { 3. Alianza estratégica } \\
\text { 4. Vigilancia }\end{array}$ \\
\hline Mover primero & $\begin{array}{l}\text { Efecto limitado, por la baja } \\
\text { frecuencia de la coopera- } \\
\text { ción (cuando ésta aumenta } \\
\text { puede apoyar la ampliación } \\
\text { de la gama) }\end{array}$ & $\begin{array}{l}\text { Puede favorecer un mover } \\
\text { primero incremental (basa- } \\
\text { do en la ampliación de la } \\
\text { gama) }\end{array}$ & $\begin{array}{l}\text { Puede favorecer un mover pri- } \\
\text { mero radical (múltiples proyec- } \\
\text { tos altamente innovadores) }\end{array}$ \\
\hline $\begin{array}{l}\text { Activos comple- } \\
\text { mentarios }\end{array}$ & $\begin{array}{l}\text { Efecto limitado, por la baja } \\
\text { complejidad de los proyec- } \\
\text { tos }\end{array}$ & $\begin{array}{l}\text { Refuerza algunos activos } \\
\text { complementarios (imagen } \\
\text { de la firma) }\end{array}$ & $\begin{array}{l}\text { Refuerza algunos activos com- } \\
\text { plementarios (imagen de la fir- } \\
\text { ma, portafolio de patentes, acti- } \\
\text { vos regulatorios) }\end{array}$ \\
\hline Patentes & $\begin{array}{l}\text { Escasa generación de pa- } \\
\text { tentes }\end{array}$ & $\begin{array}{l}\text { Generación de patentes de- } \\
\text { fensivas }\end{array}$ & $\begin{array}{l}\text { Generación de patentes ofensi- } \\
\text { vas }\end{array}$ \\
\hline
\end{tabular}

Fuente: Elaboración propia 


\section{Conclusiones}

Este trabajo analiza cómo se ve afectada la estrategia de apropiación de los resultados de las innovaciones de las firmas, cuando dichas innovaciones se basan en la CPP. El análisis se centra en los casos de nueve firmas biofarmacéuticas argentinas cuyos proyectos de innovación se desarrollan en colaboración con instituciones públicas de I+D. El trabajo plantea que la colaboración y, particularmente, la forma que ésta adopta afectan el uso y la eficacia de los distintos mecanismos de apropiación.

Los casos estudiados permiten identificar tres esquemas de colaboración (contratación, internalización y coordinación), con características distintivas y con efectos diferenciados sobre las estrategias de apropiación de las firmas. En relación a los mecanismos legales de apropiación, se observa que la cPP tiene un efecto positivo sobre los mismos e incluso que constituye una condición esencial para su generación y uso. El esquema de coordinación, al favorecer la generación de patentes ofensivas, parece ser más virtuoso respecto a los demás esquemas en este sentido. En lo referido a los mecanismos estratégicos de apropiación, la colaboración muestra diferentes efectos para cada uno de ellos. Por un lado, tiende a debilitar el uso del secreto industrial, ya que incrementa el riesgo de filtraciones hacia los competidores. Sin embargo, dentro de cada esquema, aunque especialmente en el caso de la coordinación, las firmas pueden apoyarse en diferentes elementos con el objetivo de atenuar tales riesgos. Por el otro, la colaboración genera efectos positivos tanto sobre la estrategia de mover primero como sobre el uso de algunos activos complementarios. Respecto a mover primero, se observa que los esquemas de contratación e internalización favorecen una estrategia orientada principalmente a ampliar la gama de una misma familia de productos, mientras que el esquema de coordinación permite a la firma abarcar proyectos de importante nivel de novedad. En el caso de los activos complementarios, la colaboración es un factor que contribuye a la consolidación de varios activos críticos para la firma, especialmente cuando la misma se desarrolla en el marco de los esquemas más complejos (internalización y coordinación). Dados estos efectos opuestos, donde la colaboración tanto desafía como refuerza la eficacia de los distintos mecanismos de apropiación, el esquema específico que asume la colaboración en cada caso cobra una importancia central ya que, como se ha podido apreciar, cada esquema afecta de manera diferente a los distintos mecanismos de apropiación y a su efectividad. En tal sentido, el esquema de coordinación parece ser el más funcional a la estrategia de apropiación de las firmas biofarmacéuticas analizadas.

En el marco de los resultados obtenidos en esta investigación, vale la pena destacar algunos aspectos que pueden permitir plantear nuevas preguntas de investigación a indagar. En primer lugar, la posibilidad de que el esquema de colaboración 
sea parte integrante de la estrategia de apropiación de la firma y funcione como un mecanismo de apropiación en sí mismo, con directa relevancia en la relación público-privada, al favorecer la apropiación privada respecto al partner público. En segundo lugar, dado que algunas firmas estudiadas utilizan más de un esquema de colaboración, vale la pena indagar sobre la combinación entre esquemas, en la medida en que la capacidad de combinar diversos esquemas otorga mayor solidez a la estrategia de apropiación de una firma. Por último, la firma decide caso por caso qué esquema de colaboración es más conveniente, pero, a lo largo de su trayectoria y con la evolución de sus capacidades innovadoras, evoluciona también su capacidad de cooperar y la capacidad de sofisticar su estrategia de apropiación. Se puede plantear, entonces, la existencia de un sendero evolutivo en la forma de colaboración predominante, que va desde formas más simples hacia otras más articuladas.

Si bien el presente estudio avanza sobre un tema poco estudiado por la literatura, como es el de los efectos de la CPP sobre la estrategia de apropiación de las firmas, y lo hace desde una perspectiva cualitativa que permite identificar elementos inherentes a la dinámica de la colaboración entre los partners permitiendo ir más allá de las patentes (incluso en un sector "intensivo en patentes"), el análisis podría verse enriquecido con la consideración de la perspectiva pública y con su extensión a otros sectores de actividad con características similares en sus dinámicas de innovación.

\section{REFERENCIAS BIBLIOGRÁFICAS}

Aguiar, Diego (2011), Análisis de los procesos socio técnicos de construcción de tecnologías intensivas en conocimiento en la Argentina: un abordaje desde la sociología de la tecnología sobre una empresa de biotecnología en el sector salud: el caso de Biosidus S.A. 1975-2005, tesis de doctorado, Buenos Aires, Flacso, Sede Académica Argentina.

Anlló, Guillermo; Bisang, Roberto y Stubrin, Lilia (2010), "Las empresas de biotecnología en Argentina”, Documento de Trabajo-Cepal, núm. 51.

Arrow, Kenneth (1962), "Ecoomic Welfare and the Allocation of Resources for Invention", en Richard Nelson (ed.), The Rate and Direction of Inventive Activity (pp. 609-625), Princeton, Princeton University Press.

Arundel, Anthony (2001), "The Relative Effectiveness of Patents and Secrecy for Appropriation", Research Policy, 30, pp. 611-624.

Arza, Valeria (2010), "Channels, benefits and risks of public-private interactions for knowledge transfer: conceptual framework inspired by Latin America", Science and Public Policy, 37 (7), pp. 473-484. 
Belderbos, René; Carree, Martin y Lokshin, Boris (2004), "Cooperative R\&D and firm performance”, Research Policy, 33, pp. 1477-1492.

Bercovitz, Janet y Feldman, Maryann (2007), "Fishing upstream: Firm innovation strategy and university research alliances", Research Policy, 36, pp. 930948.

Bisang, Roberto; Gutman, Graciela; Lavarello, Pablo; Sztulwark, Sebastián y Díaz, Alberto (comps.) (2006), Biotecnología y desarrollo. Un modelo para armar en la economía argentina, Buenos Aires, Prometeo y UNGS.

Bonaccorsi, Andrea y Piccaluga, Andrea (1994), "A theoretical framework for the evaluation of university-industry relationships", $R \& D$ Management, 24 (3), pp. 229-247.

Brouwer, Erik y Kleinknecht, Alfred (1999), “Innovative output and a firm's propensity to patent. An exploration of CIs micro data", Research Policy, 28, pp. 615-624.

Bureth, Antoine; Levy, Rachel; Pénin, Julien y Wolff, Sandrine (2005), "Strategic Reasons for Patenting: Between Exclusion and Coordination Rationales", Rivista di Politica Economica, 95 (5), pp. 19-46.

Caloghirou, Yannis; Aggelos, Tsakanikas y Vonortas, Nicholas (2001), "University-Industry Cooperation in the Context of the European Framework Programmes", Journal of Technology Transfer, 26 (1-2), pp. 153-161.

Carayol, Nicolas (2003), "Objectives, agreements and matching in science-industry collaborations: Reassembling the pieces of the puzzle", Research Policy, 32, pp. 887-908.

Cassier, M., (1997), "Compromis institutionnels et hybridations entre recherche publique et recherche privée", Revue d'Economie Industrielle, 79, pp. 191-212.

Cassiman, Bruno y Veugelers, Reinhilde (2002), "R\&D cooperation and spillovers: Some empirical evidence from Belgium", American Economic Review, 44 (3), pp. 1169-1184.

Cimoli, Mario y Primi, Annalisa (2008), “Technology and Intellectual Property: A Taxonomy of Contemporary Markets for Knowledge and Their Implications for Development", LEM Papers Series, pp. 1-24.

Cohen, Wesley; Nelson, Richard y Walsh, John (2000), Protecting Their Intellectual Assets: Appropriability Conditions and Why Us Manufacturing Firms Patent (or Not), Cambridge, National Bureau of Economic Research. 
D’Este, Pablo y Patel, Parimal (2007), “University-industry linkages in the UK: What are the factors determining the variety of interactions with industry?", Research Policy 36 (9), pp. 1295-1313.

Dosi, Giovanni; Marengo, Luigi y Pasquali, Corrado (2006), "How much should society fuel the greed of innovators? On the relations between appropriability, opportunities and rates of innovation", Research Policy, 35, pp. 1110-1121.

Eisenhardt, Kathleen (1989), "Building Theories from Case Study Research", Academy of Management Review, 14 (4), pp. 532-550.

Eom, Boo y Lee, Keun (2009), "Modes of Knowledge Transfer from Pros and Firm Performance: The Case of Korea", Seoul Journal of Economics, 22 (4), pp. 499-528.

Eun, Jong-Hak; Lee, Keun y Wu, Guisheng, (2006), "Explaining the 'University-run enterprises' in China: A theoretical framework for university-industry relationship in developing countries and its application to China", Research Policy, 35 (9), pp. 1329-1346.

Fernández Sánchez, Esteban; Montes Peón, José Manuel; Pérez-Bustamante, Guillermo y Vázquez Ordás, Camilo José (1998), “Acumulación, naturaleza e imitación del conocimiento tecnológico: una revisión de la literatura", Investigaciones Europeas de Dirección y Economía de la Empresa, 4 (1), pp. 11-34.

Fernández Sánchez, Esteban (2004), "Formas de apropiación de las ganancias de una innovación", Universia Business Review, primer trimestre, núm. 1, pp.70-81.

Galende del Canto, Jesús (2006), “La apropiación de los resultados de la actividad innovadora. Universidad de Salamanca”, mimeo.

González-Álvarez, Nuria y Nieto-Antolín, Mariano (2007), “Appropriability of innovation results: An empirical study in Spanish manufacturing firms", Technovation, núm. 27, pp. 280-295.

Griliches, Zvi (1990), "Patent Statistics as Economic Indicators: A Survey", Journal of Economic Literature, 28 (4), pp. 1661-1707.

Gutman, Graciela y Lavarello, Pablo (2010), "Desarrollo reciente de la moderna biotecnología en el sector de salud humana", documento del Proyecto CEUR-Conicet "Potencialidades de la biotecnología para el desarrollo industrial de Argentina", Buenos Aires. 
Hall, Bronwyn; Link, Albert y Scott, John (2001), "Barriers inhibiting industry from partnering with universities: evidence from the advanced technology program", Journal of Technology Transfer, 26, pp. 87-98.

Harabi, Najib (1995), “Appropriability of technical innovations: An empirical analysis", Research Policy, 24 (6), pp. 981-992.

Hurmelinna-Laukkanen, Pia y Puumalainen, Kaisu (2007), "Nature and Dynamics of Appropriability: Strategies for Appropriating Returns on Innovation”, $R \& D$ Management, 37 (2), pp. 95-112.

Hussinger, Katrin (2006), "Is silence golden? Patents versus secrecy at the firm level", Economics of Innovation and New Technology, 15 (8), pp. 735-752. Joly, Pierre-Benoit y Mangematin, Vincent (1996), "Profile of public laboratories, industrial partnerships and organisation of R\&D: the dynamics of industrial relationships in a large research organisation", Research Policy, 25, pp. 901-922.

Laursen, Keld, y Salter, Ammon (2006), "Open for innovation: the role of openness in explaining innovation performance among U.K. manufacturing firms", Strategic Management Journal, 27 (2), pp. 131-150.

Lee, Yong (1996), "Technology transfer and the research university: a search for the boundaries of university-industry collaboration", Research Policy, 25, pp. 843-863.

Levin, Richard; Klevorick, Alvin; Nelson, Richard; Winter, Sidney; Gilbert, Richard y Griliches, Zvi (1987), "Appropriating the Returns from Industrial Research and Development", Brookings Papers on Economic Activity, vol. 1987, núm. 3, pp. 783-831.

Levy, Rachel; Roux, Pascale y Wolff, Sandrine (2009), "An analysis of scienceindustry collaborative patterns in a large European University", The Journal of Technology Transfer, 34 (1), pp. 1-23.

Lhuillery, Stéphane y Pfister, Etienne (2009), "R\&D cooperation and failures in innovation projects: Empirical evidence from french cis data", Research Policy, 38, pp. 45-57.

Lööf, Hans y Broström, Anders (2008), "Does knowledge diffusion between university and industry increase innovativeness?", The Journal of Technology Transfer, 33 (1), pp. 73-90.

Lopez, Alberto (2008), "Determinants for R\&D cooperation: Evidence from spanish manufacturing firms", International Journal of Industrial Organization, 26, pp. 113-136. 
Mansfield, Edwin (1986), "Patents and Innovation: an empirical study", Management Science, 32 (2), pp. 173-181.

Meyer-Krahmer, Frieder y Schmoch, Urich (1998), "Science-based technologies: university-industry interactions in four fields", Research Policy, 27, pp. 835-851.

Miotti, Luis y Sachwald, Frédérique (2003), "Cooperative R\&D: why and with whom? An integrated framework of analysis", Research Policy, 32, pp. 1481-1499.

Milesi, Darío; Verre, Vladimiro; Petelski, Natalia y Aggio, Carlos (2011), “Apropiación privada de las rentas de la innovación: elementos para la discusión conceptual y el abordaje metodológico", Universidad Nacional de General Sarmiento, mimeo.

Milesi, Darío, Petelski, Natalia y Verre, Vladimiro, (2013), "Innovation and appropriation mechanisms: Evidence from Argentine microdata", Technovation, 33 (2-3), pp. 78-87.

Perkmann, M. y Walsh, K. (2007), "University-industry relationships and open innovation: Towards a research agenda", International Journal of Management Reviews, 9 (4), pp. 259-280.

(2009), "The two faces of collaboration: impacts of university-industry relations on public research", Industrial and Corporate Change, 18 (6), pp. 1033-1065.

Perkmann, Marcus; Tartari, Valentina; McKelvey, Maureen; Autio, Erkko; Broström, Anders; D’Este, Pablo; Fini, Ricardo; Geuna, Aldo; Grimaldi, Rosa; Hughes, Alan; Krabel, Stefan; Kitson, Michael; Llerena, Patrick; Lissoni, Franceso; Salter, Ammon y Sobrero, Maurizio (2013), "Academic engagement and commercialisation: A review of the literature on university-industry relations", Research Policy, 42 (2), pp. 423-442.

Ramos-Vielba, Irene y Fernández-Esquinas, Manuel (2012), "Beneath the tip of the iceberg: exploring the multiple forms of university-industry linkages", Higher Education 64 (2), pp. 237-265.

Schartinger, Doris; Rammer, Christian; Fischer, Manfred y Fröhlich, Josef, (2002), "Knowledge interactions between universities and industry in Austria: sectoral patterns and determinants", Research Policy, 31 (3), pp. 303-328.

Schumpeter, Joseph (1942), Capitalism Socialism and Democracy, Nueva York, Harper and Row. 
Teece, David (1986), "Profiting from Technological Innovation: Implications for Integration, Collaboration, Licensing and Public Policy", Research Policy, 15 (6), pp. 285-305.

Tether, Bruce (2002), "Who Co-operates for Innovation, and Why?", Research Policy, 31 (6), pp. 947-67.

Verre, Vladimiro; Petelski, Natalia y Milesi, Darío (2014), “Colaboración y estrategia de apropiación en alta tecnología: el caso de una empresa biofarmacéutica argentina", Innovar, 24 (edición especial), pp. 41-53.

Yin, Robert (1981a), "The case study as a serious research strategy", Knowledge: Creation, Diffusion, Utilization, 3, pp. 97-114. (1981b), "The case study crisis: Some answers", Administrative Science Quarterly, 26, pp. 58-65. (2003), Case study research: design and methods, 3a. ed., Applied Social Research Methods Series, vol. 5, Thousand Oaks, CA: SAgE Publications. 